\title{
Aspiration of a sponge during conscious sedation
}

\author{
Ji Young Lee, Jin Young Chon, Hyun Jung Koh, Yu Mi Ju, and Mi Ran Park \\ Department of Anesthesiology and Pain Medicine, College of Medicine, The Catholic University of Korea, Seoul, Korea
}

Conscious sedation under monitored anesthesia care is used for a wide range of operations including dacryocystorhinostomy. The most common side effects are this respiratory depression and apnea [1], which require constant care from anesthesiologists. Recently, we experienced an uncommon case of aspiration of a small surgical sponge from the nasal cavity during dacryocystorhinostomy performed on the patient under conscious sedation using the total intravenous anesthesia (TIVA) technique. This is a rare but potentially serious complication [2]. Anesthesiologists need to be cautious about maintaining adequate sedation levels to prevent adverse events from occurring. Additionally, surgeons need to ensure that surgical materials are not unintentionally misplaced or lost.

A 68-year-old woman was admitted for surgery to treat a nasolacrimal duct obstruction. After the patient arrived at the operating room, we initiated standard monitoring with bispectral index (BIS). For oxygen supplementation, a nasal cannula was placed between the lips of the patient with $\mathrm{O}_{2} 3 \mathrm{~L} / \mathrm{min}$. After confirming that all monitored parameters were normal, we infused $1000 \mathrm{mg}$ propofol (Fresopol MCT inj 2\% ${ }^{\circledR}$, Fresenius Kabi, Graz, Austria) and remifentanil (ULTIVA ${ }^{\circledR}$, GlaxoSmithKline, Brentford, Middlesex, UK) using an Orchestra ${ }^{\mathrm{TM}}$ module (Fresenius Vial, Brezins, France). By regulating the plasma remifentanil concentration to $2.0 \mathrm{ng} / \mathrm{ml}$, the BIS value of the patient fell to 85 . Next, we set the target concentration of propofol at 1.5 $\mathrm{mcg} / \mathrm{ml}$ and her BIS values were maintained at approximately 80. Under these conditions, the patient breathed spontaneously and responded to our orders. The surgeon started the operation and we maintained a stable anesthetic depth.

After 30 minutes, the patient intermittently stopped breath- ing, but responded when we attempted to reawaken her. Her BIS value was not changed between 80 to 85 in this period. We reduced the target concentration of propofol to $1.0 \mathrm{mcg} / \mathrm{ml}$ and remifentanilto $0.5 \mathrm{ng} / \mathrm{ml}$, but the patient continued to snore and took a deep breath in response to our order. About 45 minutes later, she suddenly became apneic and failed to respond to our stimuli. Her oxygen saturation decreased from 96 to $90 \%$. Even though we raised the patient's chin, her saturation decreased to $75 \%$ momently after one deep breath with BIS value also fell to 60 . We stopped the operation, and started mask ventilation with $100 \% \mathrm{O}_{2}$. Her saturation increased rapidly to $95 \%$ and the BIS value returned to 80 .

After consulting with the surgeon about continuing the operation, we intubated the patient without administering a muscle relaxant. The patient was still unresponsive despite having a BIS value of 80 . Surgeons went on operation and finished without special event. The patient fully awoke near the end of operation, she couldn't stand intubation. Therfore, we extubated unwillingly. After then, we noticed that a small sponge (Petipad ${ }^{\mathrm{TM}}$ Neurosurgical Sponges Pollak Int., Euroband, France) was missing. It had been packed into a deep nasal cavity to control bleeding but could not find it in the nasal or oral cavity. We suspected that the sponge had been swallowed rather than aspirated because the patient's vital signs remained stable with $100 \%$ oxygen saturation.

We examined the chest X-ray to determine the location of the gauze. We discovered that the radio-opaque thread dangling from the sponge was located in the midline of the chest (Fig. 1A). It followed the shape of the trachea and main bronchus. Since we had already extubated the trachea, we decided to re-

Corresponding author: Hyun Jung Koh, M.D., Ph.D., Department of Anesthesiology and Pain Medicine, Yeouido St. Mary Hospital, College of Medicine, The Catholic University of Korea, 10, 63-ro, Yeongdeungpo-gu, Seoul 150-713, Korea. Tel: 82-2-3779-1268, Fax: 82-2-783-0368, E-mail: hjkoh92@catholic.ac.kr

(c) This is an open-access article distributed under the terms of the Creative Commons Attribution Non-Commercial License (http:// creativecommons.org/licenses/by-nc/3.0/), which permits unrestricted non-commercial use, distribution, and reproduction in any medium, provided the original work is properly cited. 

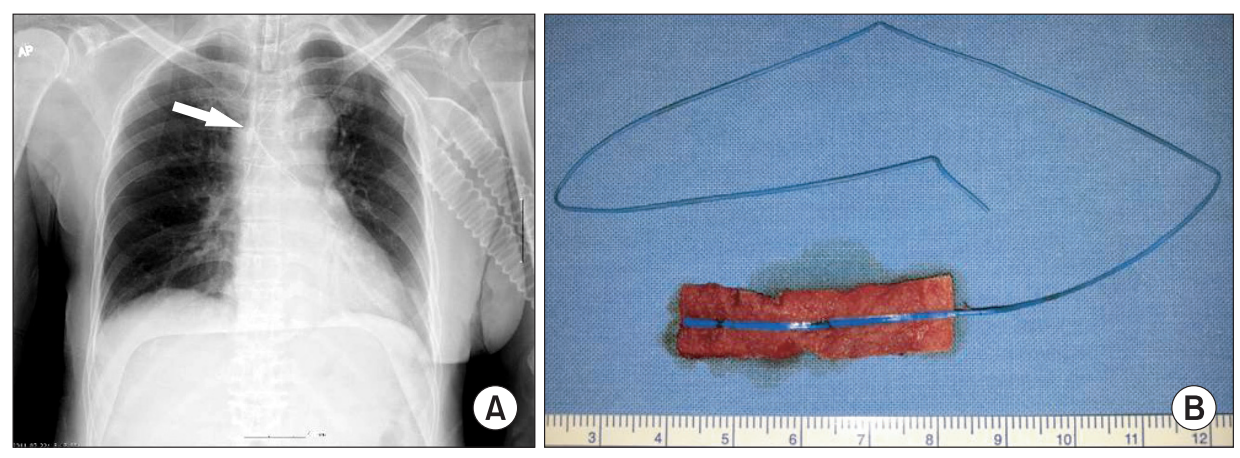

Fig. 1. (A) Pre-extubation, chest X-ray shows the probable presence of linear foreign material ( $\mathrm{R} / \mathrm{O}$ wire) within the large airway (trachea and both main bronchi). Linear atelectasis in both lower lung fields and a right mastectomy state are also seen. (B) Expectorated gauze sponge saturated with blood.

move the sponge by flexible bronchoscopy with the patient under local anesthesia. The patient started to inhale the nebulized $2 \%$ lidocaine. As soon as the patient started to inhale the cold nebulized drugs, she coughed violently and the sponge emerged (Fig. 1B). We took another chest X-ray and confirmed that the entire gauze sponge had been successfully removed. The patient was subsequently discharged without any complications.

Nowadays, TIVA with remifentanil and propofol administered by target controlled infusion is used commonly for simple and short procedures during which the patients continue to breathe spontaneously [3]. However, adverse events such as hypotension, bradycardia, respiratory depression, and desaturation (in particular, deep sedation) can occur [1]. To prevent these complication, anesthesiologists usually increase the dose or target concentrations in a slow and step-wise manner [2], and supply oxygen via various methods [4]. During an operation, it is vital that surgeons concentrate on the procedures about their performing and closely monitor the location of small items such as gauze, needles, and fragments of instruments. In the present case, an assistant who was holding the thread of the sponge lost it while we were busy maintaining the airway of the patient. The sponge was aspirated when the patient took a deep breath or during mask ventilation.

Usually, these materials are found in the esophagus rather than the bronchus or trachea [5]. During facial and oral surgery, most aspirated foreign bodies are extracted teeth and usually removed by flexible or rigid bronchoscopy. These foreign bodies sometimes can be spontaneously expectorated. In our patient, it was not certain where the sponge was until we examined the chest X-ray. In addition, the patient was comfortable, did not complain, did not cough, and had normal breathing sounds and oxygen saturation. Therefore, when there is a possibility of aspiration checking the X-ray is essential even in the absence of clinical signs.

One drawback of this case was that we extubated from the trachea before we confirmed the chest X-ray findings. Our findings highlight the importance of carefully maintaining an adequate anesthetic depth, not disturbing spontaneous ventilation, and monitoring the location of all surgical instruments at all times, especially ones that are even very small.

\section{References}

1. Agostoni M, Fanti L, Gemma M, Pasculli N, Beretta L, Testoni PA. Adverse events during monitored anesthesia care for GI endoscopy: an 8-year experience. Gastrointest Endosc 2011; 74: 266-75.

2. Xiao D, Wang F, Wang X, Tang W, Yang H, Shen S. Efficacy and security of sedation in upper gastrointestinal endoscopy in snoring patients. Zhong Nan Da Xue Xue Bao Yi Xue Ban 2010; 35: 1174-7.

3. LaPierre CD, Johnson KB, Randall BR, White JL, Egan TD. An exploration of remifentanil-propofol combinations that lead to a loss of response to esophageal instrumentation, a loss of responsiveness, and/or onset of intolerable ventilatory depression. Anesth Analg 2011; 113: 490-9.

4. Cravero JP, Beach ML, Blike GT, Gallagher SM, Hertzog JH. The incidence and nature of adverse events during pediatric sedation/ anesthesia with propofol for procedures outside the operating room: a report from the Pediatric Sedation Research Consortium. Anesth Analg 2009; 108: 795-804.

5. Shikada M, Morikawa A, Kobayashi T, Nakano T, Sakai T, Matsuda S, et al. Spontaneous expectoration of bronchial foreign body: a case report. Tokai J Exp Clin Med 2007; 32: 83-5. 